Im „Journal Club“ werden Originalarbeiten aus der internationalen Fachliteratur referiert und kommentiert.

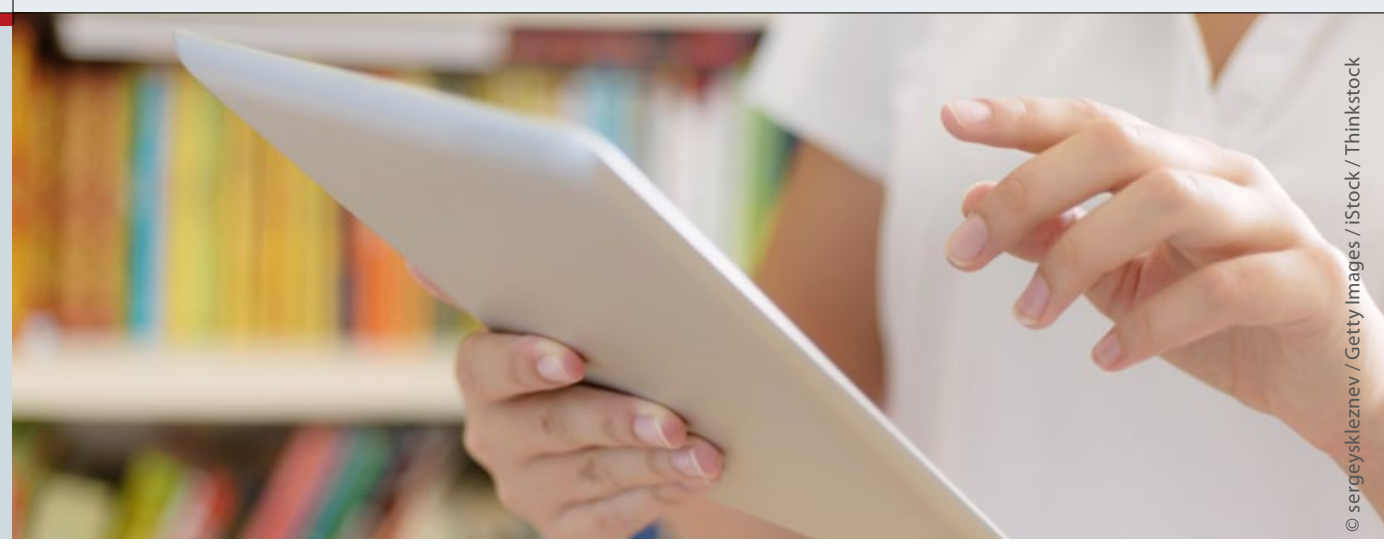

\section{Stress im Pflegeberuf: Weniger Lebensqualität und schlechtere Patientenversorgung}

\section{Beruflicher Stress kann sich ungünstig auf die physische und psychische Ge- sundheit der Berufstätigen auswirken. In einer Studie untersuchten griechi- sche Forscher, wie die hohe Belastung im Pflegeberuf die Arbeits- und die gesundheitsbezogene Lebensqualität der Pflegenden beeinflusst.}

n die Untersuchung waren 246 Pflegende (211 Frauen) mit mindestens einem Jahr Berufserfahrung eingeschlossen. Die meisten von ihnen gelten als sogenannte Pflegeassistenten (54,9\%). Dies spiegelt die griechische Pflege-Realität wider: Griechenland hat die drittniedrigste Dichte an Pflegekräften (3,3 pro 1.000 Einwohner) in den OECD-Ländern, nach der Türkei und Mexiko.

Die Teilnehmer füllten Fragebögen aus, darunter die Expanded Nursing Stress Scale (ENSS) mit Kategorien wie Umgang mit Sterbenden und Tod oder mit Patienten und Angehörigen, Probleme mit Ärzten oder mit Kollegen, der Health Survey SF-12 mit physischer und mentaler Komponente und das Caring Behaviors Inventory (CBI) mit vier Bereichen: Gewissheit mit Menschen umzugehen, professionelle/s Wissen und Fähigkeiten, Respekt gegenüber Patienten und positive Verbundenheit.

Faktoren wie Kontakt mit Tod, Patienten und ihren Angehörigen, Konflikte mit Vorgesetzten sowie Unsicherheit über die Wirksamkeit der Therapie verursachten eine signifikant erhöhte Stressbelastung. Der drittgrößte Stressfaktor waren der Studie zufolge dabei die Probleme mit dem Vorgesetzten. Dafür gaben die Autoren zwei mögliche Ursachen an: Zum einen könnte es daran liegen, dass die Vorgesetzten in Griechenland häufig in ihrer Funktion nicht gut ausgebildet sind. Zum anderen gäbe es in der griechischen Kultur einen generellen Machtkonflikt mit Obrigkeiten.

Konflikte mit Kollegen erwiesen sich als signifikante und unabhängige Prädiktoren für die CBI-Bereiche Umgang mit Menschen, professionelles Wissen sowie Respekt gegenüber Patienten. Kontroversen mit Ärzten beeinflussten zudem signifikant die CBI-Dimension Respekt gegenüber Patienten, während Auseinandersetzungen mit Vorgesetzten und Unsicherheit über die Wirksamkeit der Therapie signifikant mit dem Bereich positive Verbundenheit ungünstig zusammenhingen. Diskriminierung war negativ mit der physischen Komponente der Lebensqualität verbunden, Konflikte mit Vorgesetzten eher mit der psychischen Komponente.

Beruflicher Stress kann sich also auf die gesundheitsbezogene Lebensqualität des Pflegepersonals ungünstig auswirken, schlussfolgern die Autoren. Dies wiederum scheint die Versorgung der Patienten negativ zu beeinflussen. (aks)

Sarafis $P$ et al.: The impact of occupational stress on nurses' caring behaviors and their health related quality of life. BMC Nurs. 2016; 15:56

\section{KOMMENTAR}

Keine Frage, unser Beruf ist stressig. Wir müssen hohen Anforderungen nachkommen, haben viel Verantwortung und zu wenig Autorität. Es ist bekannt und durch
Studien belegt, dass sich die extreme Belastung negativ auf unsere physische und psychische Gesundheit auswirkt. Durch arbeitsbezogenen Stress unterlaufen uns Fehler und wir haben weniger Mitgefühl für die Patienten - die pflegerische Qualität leidet.

Der Kontakt mit dem Tod ist auf einer Intensivstation unvermeidbar. Manche Patienten sterben am Tag ihrer einlieferung, andere erliegen ihrer Erkrankung erst nach ein paar Tagen oder Wochen. Gerade der Tod von Patienten, die man über einen längeren Zeitraum betreut hat, geht nicht spurlos an uns vorüber. Auch die Angehörigen verlangen so einiges von den Pflegekräften ab. Sie haben viele Fragen und verstehen oft nicht, wie schlecht es dem Patienten wirklich geht Jeden Tag beantwortet man als Pflegende ähnliche Fragen und erfüllt Wünsche wie: "Ich glaube mein Vater möchte anders liegen", "Meine Mutter hat Durst" oder "Könnten Sie bitte ein anderes Kopfkissen besorgen?".

Mit einem guten Team und in professioneller Zusammenarbeit mit den Ärzten kann man den Stress, den wir durch die Betreuung der Patienten und deren Angehörige haben, kompensieren. Doch wenn es Konflikte mit den Kollegen und im interprofessionellen Team gibt, schadet das unserer physischen und psychischen Gesundheit. Wir sind angespannt und haben weniger Geduld und Empathie für die Wünsche und Bedürfnisse der Patienten und Angehörigen. Die vorgestellte Studie kann uns und unseren Vorgesetzten helfen Maßnahmen zu finden, die die Stressoren minimieren.

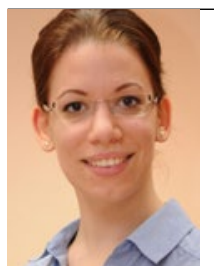

Johanna Kurze GuKP für Intensivpflege Helios Klinikum Emil von Behring Nordsternstraße 2 10825 Berlin 